\title{
Short Range Gravity Potential Self Energy and Nonlinear Potential Dependent Lorentz Transformation
}

\author{
Ali Sulaiman Mohamed ${ }^{1}$, M. Dirar ${ }^{1}$, Ahmed Elhassan Elfaki ${ }^{1}$, Rawia Abdelgani Elobaid ${ }^{1}$, \\ Amel Abdallah Ahmed Elfaki ${ }^{1}$, Bashir Elhaj Ahmed ${ }^{2}$, Abdelnabi A. Elamin ${ }^{2}$, M. Alhilo', \\ Fatma Madani ${ }^{3}$
}

${ }^{1}$ Department of Physics, College of Science Sudan University of Science \& Technology, Khartoum, Sudan

${ }^{2}$ Department of Physics, Faculty of Science and Technology, Omdurman Islamic University, Omdurman, Sudan

${ }^{3}$ Department of Physics, College of Applied Medical Science, Taif University of, Taif, KSA

\section{Email address:}

alisuleman66@yahoo.com (A. S. Mohamed), aelamain@yahoo.com (A. A. Elamin)

\section{To cite this article:}

Ali Sulaiman Mohamed, M. Dirar, Ahmed Elhassan Elfaki, Rawia Abdelgani Elobaid, Amel Abdallah Ahmed Elfaki, Bashir Elhaj Ahmed, Abdelnabi A. Elamin, M. Alhilo, Fatma Madani. Short Range Gravity Potential Self Energy and Nonlinear Potential Dependent Lorentz Transformation. American Journal of Modern Physics. Vol. 6, No. 2, 2017, pp. 32-36. doi: 10.11648/j.ajmp.20170602.12

Received: February 1, 2017; Accepted: February 24, 2017; Published: April 11, 2017

\begin{abstract}
Using Poisson equation beside Maxwell distribution law for very hot star core consisting of elementary particles one can find new Poisson equation. This equation predicts existence of short range gravity force. This short force may have a link with short range nuclear force, thus raises a hope in unifying gravity and nuclear force. This short range field beside long range field secures singular finite self-energy. This central role of potential in unifying self-energy for high relativistic particles at star cores requires seeking Lorentz transformation that accounts for effect of fields. This work derived new Lorentz transformation for accelerated frame. This transformation is potential dependent and reduces to SR and explains time dilation and gravitational red shift.
\end{abstract}

Keywords: Short Range Gravity, Nonlinear Potential, Maxwell Distribution Law, Poison Equation

\section{Introduction}

The history of gravitational field dates starts from the Newton inverse square law of gravitation [1]. This law explains successfully the motion of freely falling objects towards the earth surface. It is also explains some a astronomical phenomenon like the motion of planets around the sun, beside the motion of the moon and satellite around the earth. The satellite of the planets and the satellites in their orbits attributed.

To centrifugal force which counters balance the gravitational attractive force. The motion of astronomical objects is assumed to be inside hypothetical fluid called ether [2]. The effect of earth motion on the speed of light, which was studied by Mickelson and Morley, shows that no ether no ether exist and shows also that the speed of light is a universal constant. This is since the speed of light is not affected or changed by the motion of the source or the observer or both. This fact in direct conflict with Newton's law, especially the law of velocity addition the Galilean transformation [3]. This conflict was removed by the famous Einstein theory of special relativity (SR) which uses Lorentz transformation instead of Galilean transformation. This SR changes radically the motion of space and time. According to SR time interval and distance depend on the relative motion of the observer with respect to the mass energy. Einstein SR succeeded in explaining a wide verity of physical phenomena. It explains photoelectric effect, Compton Effect, pair production, Time dilatation in meson decay, beside more physical observations [4].

Later on Einstein generalizes SR to the so called general relativity (GR) to explain the gravitational phenomena $[5,6]$. It assumes that the gravity results from the space bending made by matter [7]. Einstein GR succeeded in explaining most of gravitational phenomena [7]. Namely the so called big bang (BB) cosmological model explain some important cosmological phenomena like universe expansion, existence of relic microwave back ground and galaxy formation, beside 
stars evaluation filed is measured by the optional $\varphi$. This potential is defined as the work done to bring a unit mass from infinity to a certain point. According to the Newton law this potential affected by the matter density $\rho$.

Einstein special relativity (SR) and general relativity (GR) are one of the big a achievement in physics. Special relativity $[1,2]$ is concerned with describing the behavior of high speed particles of macro and micro world.

Unfortunately SR suffers from noticeable setbacks. First of all does not reduce to Newton one, since its energy expression for small velocity does not recognize potential term [3]. Also SR in general does not have any term which is sensitive to field which is in direct conflict with the physics mean stream $[8,9,10]$. General relativity which partially solves this problem for gravitational field, successfully describes many astronomical and cosmological phenomena $[7,8]$. However GR gives singular solutions for massive and super massive objects $[11,12]$

This means that GR predicts its own break down in these application [11, 12] different attempts were made to cure these defects. The so called generalized special relativity think that still SR is valid by generalizing Lorentz transformation in the so called generalized SR (GSR)[13, 14]. Some authors also try to cure singularity problem by suggesting repulsive gravity force [15, 16, and 17]. But they are not based on Poisson equation or Maxwell statistical distribution. One thus needs a new model which cures some of these defects. This is done in section (2), which is concerned with non singular model and section (3) which is devoted for potential dependent Lorentz transformation. Section (4) and (5) are concerned with discussion and conclusion.

\section{Short Range Gravity Field}

Poisson Newton equation states that matter density $\rho$ generates gravity field of potential per unit mass $\emptyset$. according to the equation

$$
\begin{gathered}
\nabla^{2} \emptyset=4 \pi G \rho=4 \pi G m n_{0} e^{-\beta_{0} \emptyset}=(1-\beta \emptyset) \mathrm{C}_{0} \\
C_{0}=4 \pi G \rho
\end{gathered}
$$

$$
\left(\gamma^{2} r^{n+2}+\gamma(n+2) r^{n+1}+n(n+1) r^{n}+n \gamma r^{n+1}\right) e^{\gamma r}=c_{2} r^{n+2} e^{\gamma r}
$$

Eliminating $e^{\gamma r}$ from both sides of equation (13) yields:

$$
\gamma^{2}+\frac{\gamma(n+2)}{r}+\frac{n(n+1)}{r^{2}}+\frac{n \gamma}{r}=c_{2}
$$

For $n=-1$

$$
\begin{gathered}
\gamma^{2}+\frac{\gamma(-1+2)}{r}+\frac{-1(-1+1)}{r^{2}}+\frac{-\gamma}{r}=c_{2} \\
\gamma^{2}=c_{2}
\end{gathered}
$$

Or

$$
\gamma= \pm \sqrt{c_{2}}
$$

Where the particle density obeys Maxwell's equations:

Consider the behavior of elementary particles which form very hot gas at the star core. This meaning that:

$$
\begin{gathered}
\mathrm{m}_{0} \ll 1 \beta(\mathrm{kT})^{-1} \ll 1 \text { therefore } \mathrm{x}=\beta_{0} \varphi \ll 1 \\
n=n_{0} e^{-\beta E}=n_{0} e^{-\beta m_{0} \emptyset}=n_{0} e^{-\beta_{0} \emptyset} \approx n_{0}\left(1-\beta_{0} \varnothing\right)
\end{gathered}
$$

For spherically symmetric field

$$
\frac{1}{r^{2}} \frac{\partial}{\partial r}\left(r^{2} \frac{\partial \emptyset}{\partial r}\right)=\left(1-\beta_{0} \emptyset\right) C_{0}
$$

To simplify this equation let us define the function $f$ to be

$$
\mathrm{f}=\left(1-\beta_{0} \emptyset\right) C_{0}
$$

Consider solution of the form

$$
f=r^{n} e^{\gamma r}
$$

From equation (3)

$$
\begin{array}{r}
d f=-\beta d \emptyset \\
d \emptyset=-\frac{1}{\beta_{1}} \mathrm{df}
\end{array}
$$

Where $\beta_{1}=C_{0} \beta_{0}$

Let

$$
\beta_{1}=-C_{2}
$$

Divide equation (5) both side by dr to get

$$
\begin{gathered}
\frac{d \emptyset}{d r}=\frac{1}{c_{2}} \frac{d f}{d r} \\
\frac{d f}{d r}=n r^{n-1} e^{\gamma r}+\gamma r^{n} e^{\gamma r}
\end{gathered}
$$

Hence inserting eq,s (9), (8) and (3) in (2) yields

$$
\begin{aligned}
& \frac{1}{c_{2}} \frac{1}{r^{2}} \frac{\partial}{\partial r}\left(r^{2}\left(n r^{n-1} e^{\gamma r}+\gamma r^{n} e^{\gamma r}\right)\right)=f \\
& \frac{1}{c_{2}} \frac{1}{r^{2}} \frac{\partial}{\partial r}\left(\gamma r^{n+2}+n r^{n+1}\right) e^{\gamma r}=r^{n} e^{\gamma r}
\end{aligned}
$$

Multiply both sides by $r^{2} c_{2}$

$$
\frac{\partial}{\partial r}\left(\gamma r^{n+2}+n r^{n+1}\right) e^{\gamma r}=c_{2} r^{n+2} e^{\gamma r}
$$$$
\text { Therefore } \mathrm{f} \text { can be written according to (4) and (16) as: }
$$

$$
f=\frac{1}{r} e^{+\sqrt{c_{2}} r}
$$

According to equations (1),(2) and (7) equation (17)reads

$$
\begin{gathered}
\mathrm{C}_{0}-\mathrm{C}_{0} \beta_{0} \emptyset=\frac{1}{\mathrm{r}} \mathrm{e}^{-\sqrt{4 \pi G m n_{0} \beta_{0}} \mathrm{r}} \\
\varnothing=\frac{1}{\beta_{0}}-\frac{1}{C_{0} \beta_{0} r} e^{-\beta_{1} r}=\frac{1}{\beta_{0}}\left(1-\frac{1}{4 \pi G m n_{0}} e^{-\sqrt{4 \pi G m n_{0} \beta_{0}} r}\right)
\end{gathered}
$$

Assuming the existence of attractive long range field beside the short one, the total potential is given by 


$$
\emptyset=\emptyset_{S}+\emptyset_{L}=\frac{1}{\beta_{0}}\left(1-\frac{1}{C_{0} r} e^{-\beta_{r} r}\right)-\frac{G m}{r}
$$

Consider now the region near the center of mass $(r \rightarrow 0)$
Thus:

Hence

$$
\varnothing=\frac{1}{\beta_{0}}\left(1-\frac{1}{C_{0} r}\left(1-\beta_{1} r\right)\right)-\frac{G m}{r}=\frac{1}{\beta_{0}}-\frac{1}{\beta_{0} C_{0} r}+\frac{\beta_{1}}{C_{0}}-\frac{G m}{r}
$$

The finiteness of $\emptyset$ requires

$$
\beta_{0} C_{0}=-\frac{1}{G m}
$$

In this case

$$
\varnothing=\emptyset_{0}=\frac{1}{\beta_{0}}+\frac{\beta_{1}}{C_{0}}
$$

Thus the self energy, at which energy is a minimum, is given by

$$
\mathrm{E}=\mathrm{m}_{0} \emptyset_{0}=\mathrm{m}_{0}\left(\frac{1}{\beta_{0}}+\frac{\beta_{1}}{\mathrm{C}_{0}}\right)
$$

This means that when $r$ is very small the potential is finite, and no singularity exists. The minimum $\mathrm{r}$ can be obtained from equation (19) to get

$$
\begin{gathered}
\frac{1}{\beta_{0} C_{0} r^{2}} e^{-\beta 1 r}+\frac{\beta_{1}}{\beta_{0} C_{0} r} e^{-\beta 1 r}+\frac{G}{r^{2}}=0 \\
\left(1+\beta_{1} r\right) e^{-\beta 1 r}+C_{1} \beta_{0} C_{0}=0
\end{gathered}
$$

For small $\mathrm{r}$

$$
\begin{gathered}
\left(1+\beta_{1} r\right)\left(1-\beta_{1} r\right)+C_{0} \beta_{0} G=0 \\
1-\beta_{1}^{2} r^{2}=C_{0} \beta_{0} G \\
r=\sqrt{\frac{1-C_{0} \beta_{0} G}{\beta_{1}^{2}}}
\end{gathered}
$$
is

Thus $\mathrm{r}$ is real when $C_{0} \beta_{0} G<1$ this means that critical mass

$$
\mathrm{M}<\frac{\mathrm{R}^{\frac{3}{2}}}{\mathrm{G}(3 \beta)^{\frac{3}{2}}} \mathrm{M} \beta(4 \pi \mathrm{G} \rho) \mathrm{G}<1
$$

This means that the star mass should be less than a certain critical mass Mc, i.e.

$$
M<M_{c}
$$

Where

$$
M_{c}=\frac{R^{\frac{3}{2}}}{G(3 \beta)^{\frac{3}{2}}}
$$

Since self energy requires $r \rightarrow 0$. Thus it is quite natural to expect $M$ to be small and less than certain critical value

\section{Nonlinear Potential Dependent Lorentz Transformation}

The Principle of Special Relativity states that the laws of nature are invariant

Under a particular group of space-time coordinate transformations, called Lorentz transformations. A Lorentz transformation is a transformation from one system of spacetime coordinates $\mathrm{S}$ to another systemŚ. Let us derive a new coordinate transformation from one inertial system to another, which replaces the Galilean transformation, on the basis of Einstein's two postulates for the special theory of relativity. For this purpose, we consider two inertial systems $\mathrm{S}(\mathrm{x}, \mathrm{y}, \mathrm{z}, \mathrm{t})$ and $\mathrm{S}\left(\mathrm{x}^{\prime}, \mathrm{y}^{\prime}, \mathrm{z}^{\prime}, \mathrm{t}^{\prime}\right)$. The inertial system $\mathrm{S}^{\prime}$ is assumed to be uniformly moving in the $\mathrm{x}$ direction in a potential field $\emptyset$ with velocity $\mathrm{v}$ relative to the inertial system $\mathrm{S}$, keeping each coordinate axis parallel to the corresponding axis of the latter. Now, let an event occur at the position (x, y, $\mathrm{z}$ ) at the time $\mathrm{t}$ in the inertial system $\mathrm{S}$, and let the same event occur at the corresponding position $\left(x, y^{\prime}, z^{\prime}\right)$ and at the corresponding time $\mathrm{t}^{\prime}$ in the inertial system $\mathrm{S}$. Then, one needs to find how the sets of space-time coordinates, $(\mathrm{x}, \mathrm{y}, \mathrm{z}$, t) and $\left(x, y^{\prime}, z, t^{\prime}\right)$ are transformed to each other under the two postulates for the special theory of relativity.

The distance between the origin of two axis is given by L, where

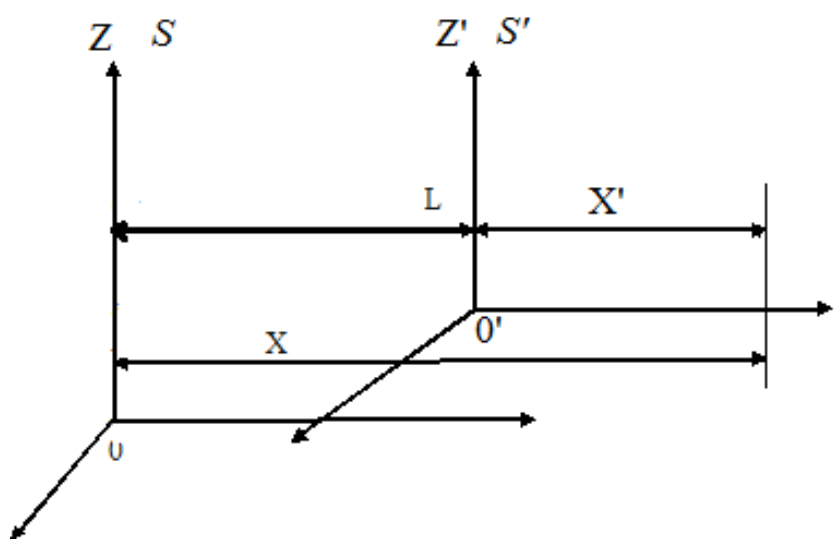

Figure 1. Shows two inertial systems $S$ and $S$.

For motion with constant acceleration

$$
L=v_{0} t+\frac{1}{2} a t^{2}
$$

The velocity at instant of time is given by

$$
v=v_{0}+a t
$$

By adding and subtracting $\frac{1}{2} a t^{2}$ for equation (24) we get 


$$
\begin{gathered}
L=v_{0} t+\frac{1}{2} a t^{2}-\frac{1}{2} a t^{2}+\frac{1}{2} a t^{2}=v_{0} t+a t^{2}-\frac{1}{2} a t^{2} \\
=\left(v_{0}+a t\right) \mathrm{t}-\frac{1}{2} a t^{2}
\end{gathered}
$$

From equation (25) this quantity $v_{0}+a t$ equal to $\mathrm{v}$

$$
L=v t-\frac{1}{2} a t^{2}
$$

One can write $\mathrm{L}$ in terms of the potential per unit mass, to be in the form

$$
L=v t-\frac{1}{2 x} a x t^{2}=v t+\frac{\emptyset}{2 x} t^{2}
$$

Where $\varnothing=-a x$

Consider now the nonlinear Lorentz transformation, in time of the form

$$
\begin{gathered}
x=\gamma(\dot{x}+\dot{L}) \\
x=\gamma\left(\dot{x}+v \dot{t}+\frac{\emptyset}{2 \dot{x}} \dot{t}^{2}\right)
\end{gathered}
$$

Where $\mathrm{x}$ is the position of event in $\mathrm{S}$. Thus the equivalent position in $S^{\prime}$ is given by

$$
\begin{gathered}
\dot{x}=\gamma(x-L) \\
\dot{x}=\gamma\left(x-v t-\frac{\emptyset}{2 x} t^{2}\right)
\end{gathered}
$$

Consider a pulse of light emitted when the origins of $\mathrm{S}$ and $S$ coincide at $\mathrm{t}=\dot{t}=0$

Thus $\mathrm{x}=\mathrm{ct}$ and $x^{\prime}=\mathrm{c} \dot{t}$. Substitute these quantities in equations (31) \& (32) respectively gives

$$
\begin{aligned}
c t & =\gamma\left(c \dot{t}+v \dot{t}+\frac{\emptyset}{2 c t} \dot{t}^{2}\right) \\
& =\gamma\left(c+v+\frac{\emptyset}{2 c}\right) \dot{t} \\
& =\gamma c\left(1+\frac{v}{c}+\frac{\emptyset}{2 c^{2}}\right) \dot{t}
\end{aligned}
$$

From which we get $\mathrm{t}$ as

$$
t=\gamma\left(1+\frac{v}{c}+\frac{\varnothing}{2 c^{2}}\right) t
$$

And from equation (28)

$$
\begin{gathered}
c \dot{t}=\gamma\left(c t-v t-\frac{\emptyset}{2 c t} t^{2}\right) \\
=\gamma\left(c t-v t-\frac{\emptyset}{2 c} t\right)=\gamma c\left(1-\frac{v}{c}-\frac{\emptyset}{2 c^{2}}\right) t \\
\dot{t}=\gamma\left(1-\frac{v}{c}-\frac{\emptyset}{2 c^{2}}\right) t
\end{gathered}
$$

Substitute equation (36) in to equation (39) yields

$$
\begin{gathered}
t=\gamma\left(1+\frac{v}{c}+\frac{\emptyset}{2 c^{2}}\right) \cdot \gamma\left(1-\frac{v}{c}-\frac{\emptyset}{2 c^{2}}\right) \dot{t} \\
1=\gamma^{2}\left(1+\frac{v}{c}+\frac{\emptyset}{2 c^{2}}\right)\left(1-\frac{v}{c}-\frac{\emptyset}{2 c^{2}}\right) \\
\gamma^{-2}=\left(1+\frac{v}{c}+\frac{\emptyset}{2 c^{2}}\right)\left(1-\frac{v}{c}-\frac{\emptyset}{2 c^{2}}\right)
\end{gathered}
$$

This expiration represents potential and speed dependent Lorentz's transformation

From the fact that $(1+f)(1-f)=1-f^{2}$

Where $f=\left(\frac{v}{c}+\frac{\emptyset}{2 c^{2}}\right)$ yields

$$
\gamma^{-1}=\left(1-\left(\frac{v}{c}-\frac{\emptyset}{2 c^{2}}\right)^{2}\right)^{\frac{1}{2}}
$$

$$
\begin{gathered}
\gamma^{-1}=\left(1-\left(\frac{v^{2}}{c^{2}}-2 \frac{v \emptyset}{2 c^{3}}+\frac{\emptyset^{2}}{4 c^{4}}\right)\right)^{\frac{1}{2}} \\
\gamma^{-1}=\left(1-\left(\frac{v^{2}}{c^{2}}-\frac{v \emptyset}{c^{3}}+\frac{\emptyset^{2}}{4 c^{4}}\right)\right)^{\frac{1}{2}}
\end{gathered}
$$

$$
\begin{aligned}
& \emptyset=\frac{V}{m} \text { or } V=m \text { and } V=-\int F d x \\
& \mathrm{~V}=-\int F d x=-\int \operatorname{mad} x=-m a \int d x=-\max
\end{aligned}
$$

From which we conclude that $\emptyset=-a x$

Let us now see how this new Lorentz transformation related to SR one. For $\emptyset=0$ clearly equation (41).

$$
\gamma^{-1}=\left(1-\frac{v^{2}}{c^{2}}\right)^{\frac{1}{2}}
$$

Thus it reduces to Einstein SR when no potential exist one needs also to see its relation within general relativity predictions.

Consider the case when

$$
\frac{\emptyset}{2 c^{2}} \ll \frac{v}{c}
$$

Thus equation (39) gives

$\gamma^{-1}=\left(1-\left(\frac{v}{C}\right)^{2}\left(1-\frac{v \emptyset}{2 C}\right)^{2}\right)^{\frac{1}{2}}=\left[1-\left(\frac{v}{C}\right)^{2}\left(1-\frac{v \emptyset}{C}\right)\right]^{\frac{1}{2}}$

For particle moving from rest

$$
v^{2}=v_{0}^{2}+2 a x=2 a x=-2 \varnothing
$$

Thus

$$
\gamma=\left[1+\frac{2 \emptyset}{C^{2}}\left(1-\frac{v \emptyset}{C}\right)\right]^{\frac{-1}{2}} \approx\left[1+\frac{2 \emptyset}{C^{2}}\right]^{\frac{-1}{2}}
$$

According to this relation

$$
t=\gamma \dot{t}=\gamma t_{0}=\left[1+\frac{2 \varnothing}{C^{2}}\right]^{\frac{-1}{2}} t_{0}
$$

This equation resembles the general relativistic time dilation relation which was verified experimentally.

\section{Discussion}

Using Poisson equation (1), beside Maxwell distribution law in equation (2), one can find new Poisson equation. For very small $\beta_{0}\left(\beta_{0} \sim(k T)^{-1}\right.$, i.e. for very high temperature, a short range gravity field exist see equation (18). This is 
agrees with the assumptions that such short range field is observed near the stars cores, where the temperature is very high, to prevent collapse. According to equation (19), when one assumes the existence of attractive force, beside the short range one, the potential $\emptyset$ is constant and finite at the center of mass. This means that the stars and elementary particles have finite self-mass (see equation 23). thus this model is more advanced than that SR. The relativistic behavior of particles in this short or long range field can also be found by seeking Lorentz transformation that accounts for the effect of fields. This is done by equations (31) and (32). Assuming the speed of light to be constant. One find the Lorentz transform (43) which depends on speed $\mathrm{v}$ and potential $\varnothing[$ (see equation (43)]. surprisingly equation (43) reduces to SR one for no potential and predict general relativistic time dilation for weak field [ see equation (47), (52)].

The capability of this model in predicting finite self mass and it is agreement with wide experimental observation shows that this version of SR is still capable of describing of physical phenomena

\section{Conclusion}

Using Newton Poisson equation beside Maxwell statistical distribution law one can predict existence of short range repulsive gravity force. This gravity force may have a link with strong nuclear force and predicts finite self mass energy and help in constructing non singular model for stars and universe. It also enables using describing non singular and non collapsing black holes. By using Newton's second law for constant acceleration one can drive new Lorentz transformation which recognizes the effect of potential as well as velocity. This reduces to that SR beside predicting the gravity time dilation.

\section{References}

[1] M. Born, Einstein's theory of relativity, Dover, 1962.

[2] T. M. Hellwill, Introduction to special relativity, Allyn and Bacon, 1966.

[3] E. P. Ney, Electromagnetism and Relativity, Harper and Row, 1962.

[4] David Hlliday, Robert Resnick physics (part $1 \& 2$ combined)
Third edition John Wiley and sons, New York, 1978.

[5] Steven Weinberg, Gravitation and cosmology, John Wiley and sons, VSA, 1972.

[6] Alan. P. Wightman, William H. press, Richard H. price, Soul A. Teukolsky, Problem book in relativity and gravitation, Princeton University press, Princeton, New Jersey, 1979.

[7] R. Adler, M. Bazir, Schiffer, Introduction to general relativity, Mc Graw Hill, New York (1960).

[8] M. Dirar et al, Neutrino speed can exceed the speed of light within the frame work of generalized special relativity and Savickas model, Natural Science vo.15, No.6 685-688(2013).

[9] D. Savicks, relation between Newtonian mechanics, general relativity and quantum mechanics, Am. J. Phy 70(8) (august 2002).

[10] M. Dirar, K. G Elgaylani, K. M. H, M. H. E, M. A. A, A. E. T, F. M, department of Einstein Generalized Special Relativity using Lorantz transformation, international. J of science and research (IJSR) V.3, ISS (6), June 2014, on line: 2319-7064.

[11] A. A Longunov, Gravitation and elementary particles physics, mir Publisher Moscow (1983).

[12] M. Dirar, A. E. T, M. H. S, A Generalizing field cosmology, modern physics letters A, vo.13, No.37,(1998) 3025-3031.

[13] Black hole on the basic of generalized special relativity, Vol.3, ISS (01), Jan 2017 ISSN: 2395.3470 M. Dirar, Hashim, M. Sawsan. A. E, generalized the general relativity using generalized Lorantz transformation, international. J. of innovative science, Engineering a Technology, V. 3, IS (4), April 2016, ISSN2348-7968.

[14] M. Dirar, A. Elnabi, Asma. M. E, Rawia. A. E, Fatima Medani and Hassab E. G, the effect of speed and potential on time, mass and energy on the basic of newton and relativity prediction, international. $\mathrm{J}$ of engineering science and management, Abdalla, 6(1), January-march 2016.

[15] M. Dirar, Mahmmed. S. D, Ahmed A. Elfaki, Sawsan A. E, stars Evolution, start self energy and nonsingular.

[16] M. Dirar, M. S. D, Ahmed Elfaki, Sawsan. A. E, Equilibrium of stars within the frame work of generalized special relativity theory, international. $\mathrm{J}$ of innovative science, Engineering Technology, V.4, ISS (1), Jan 2017, ISSN2348 7698(online).

[17] Hashim. M. Ali, M. Dirar, Fatima Medani + sawsan. A. E, Matter + Antimatter generation + repulsive Gravity force, international. $\mathrm{j}$ of scientific engineering and Applied science (IJSEAS), vol.2, ISS(5), May 2016,ISSN:2395-3470. 